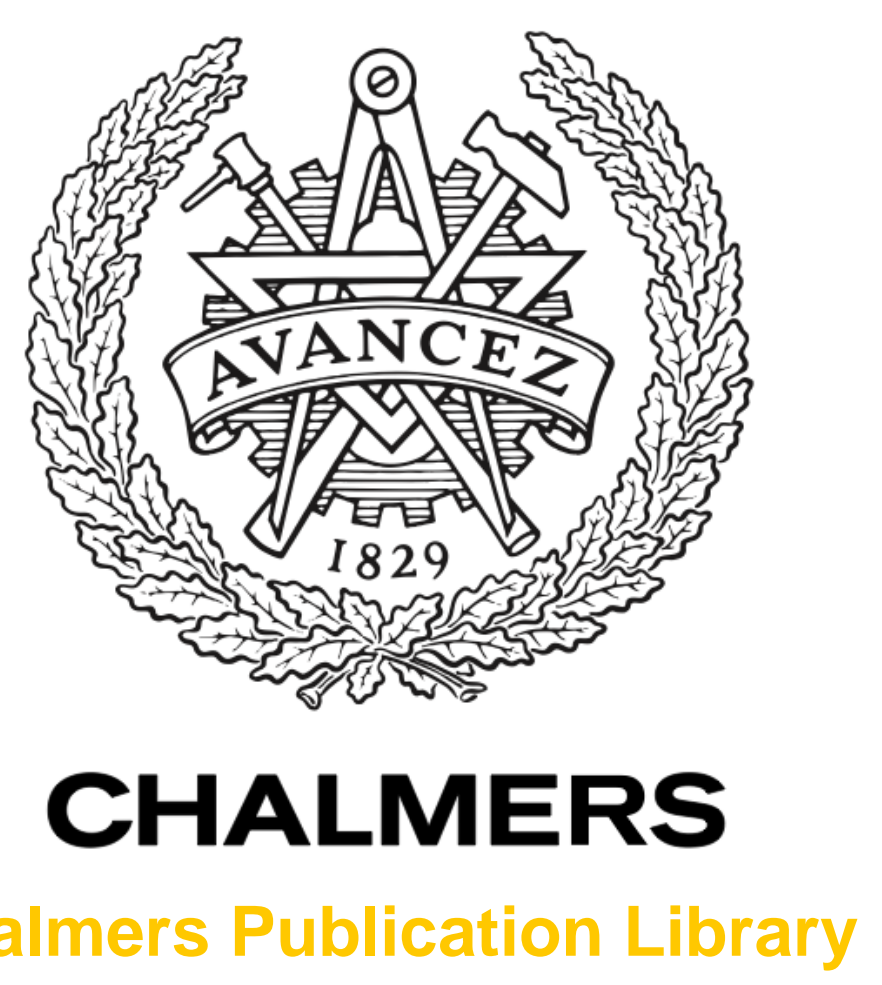

Chalmers Publication Library

\title{
A Quadraxial Feed for Ultra-Wide Bandwidth Quadruple-Ridged Flared Horn Antennas
}

This document has been downloaded from Chalmers Publication Library $(\mathrm{CPL})$. It is the author's version of a work that was accepted for publication in:

8th European Conference on Antennas and Propagation, EuCAP 2014, The Hague, The Netherlands 6-11 April 2014

Citation for the published paper:

Beukman, T. ; Ivashina, M. ; Maaskant, R. (2014) "A Quadraxial Feed for Ultra-Wide Bandwidth Quadruple-Ridged Flared Horn Antennas". 8th European Conference on Antennas and Propagation, EuCAP 2014, The Hague, The Netherlands 6-11 April 2014 pp. $3312-3316$.

http://dx.doi.org/10.1109/EuCAP.2014.6902537

Downloaded from: http://publications.lib.chalmers.se/publication/203787

Notice: Changes introduced as a result of publishing processes such as copy-editing and formatting may not be reflected in this document. For a definitive version of this work, please refer to the published source. Please note that access to the published version might require a subscription. 


\title{
A Quadraxial Feed for Ultra-Wide Bandwidth Quadruple-Ridged Flared Horn Antennas
}

\author{
Theunis S. Beukman ${ }^{1}$, Marianna V. Ivashina ${ }^{2}$, Rob Maaskant ${ }^{2}$, Petrie Meyer ${ }^{1}$, Carlo Bencivenni ${ }^{2}$ \\ ${ }^{1}$ Department of Electrical and Electronic Engineering, Stellenbosch University, Stellenbosch, South Africa \\ Email: theunis.beukman@gmail.com; pmeyer@sun.ac.za \\ ${ }^{2}$ Department of Signals and Systems, Chalmers University of Technology, Gothenburg, Sweden \\ Email: marianna.ivashina@chalmers.se; rob.maaskant@chalmers.se; carlo.bencivenni@chalmers.se
}

\begin{abstract}
A quadraxial feed, excited by two orthogonal differential modes, is proposed for ultra wideband quadruple-ridged flared horn (QRFH) antennas that obviates the need for a balun. It is shown that in this configuration the fundamental $\mathrm{TE}_{11}$ mode is most strongly excited over the entire frequency band, while the higher-order modes are significantly suppressed, as compared to the conventional excitation using the ridge-to-coax balun transition. These properties lead to several advantages for QRFH antenna applications which require frequency-invariant beam characteristics, high port isolation and low cross-polarisation level, such as e.g. reflector antenna feeds for future radio telescopes.
\end{abstract}

Index Terms-ultra wideband antennas, reflector antenna feeds, quadruple-ridged waveguides, radio astronomy.

\section{INTRODUCTION}

Balanced excitations are required to achieve ultra wideband performance with the novel reflector-antenna feeds consisting of pair(s) of interleaved spiral, log-periodical or other travelling-wave structures, which are currently being developed for radio astronomy instruments [1]-[4]. This excitation is commonly realised by using a balun interfacing the balanced antenna to single-ended amplifiers. However, practical designs of such passive networks are often bulky and lead to power dissipation losses. Power dissipation reduces the antenna radiation efficiency and increases the system thermal noise temperature [5]. Another limiting factor is that the ultra wideband balun design restricts the reference impedance for the optimum noise matching between the antennas and low-noise amplifiers (LNAs). To obviate these disadvantages, differential low-noise amplifiers (dLNA), which can be directly integrated at the antenna terminals, represent an interesting alternative solution.

In this paper a new type of differential feed is introduced for the integration of dLNAs with the quadruple-ridged flared horn (QRFH) antenna shown in Fig. 1. A cross-section of the proposed feed design is shown in Fig. 2(a). This feed suppresses the high-order waveguide modes, while strongly exciting the desired fundamental mode across a wide frequency band.

\section{Feed Description And Design Procedure}

The proposed feed for the QRFH antenna, as shown in Fig. 2(a), consists of a quadraxial line with each separate

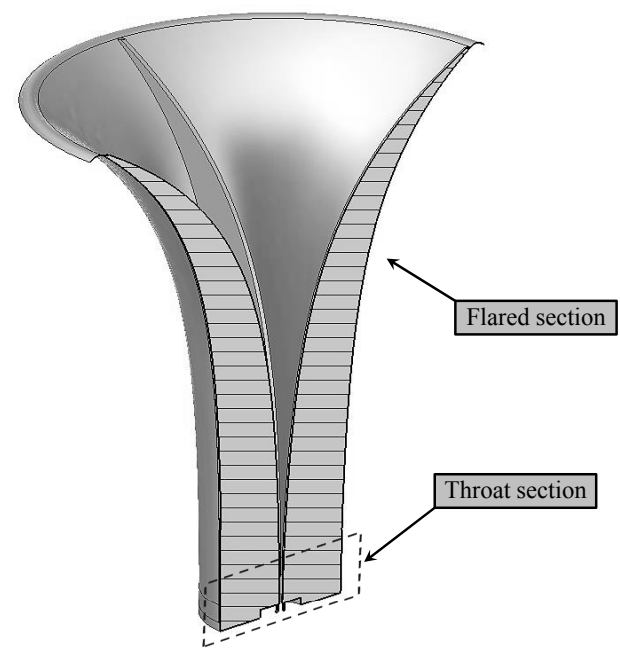

Fig. 1. CAD view of a cross-section of the QRFH antenna with the quadraxial feed.

centre conductor connected to a corresponding ridge. The quadraxial line feeds through a cylinder in the back lid of the QRFH as illustrated in the inset of Fig. 2(a). The centre conductors are differentially excited in pairs to form two twinaxial transmission lines.

This feeding technique allows the excitation of two orthogonal $T E_{11}$ fundamental modes in the ridged waveguide that are required for the dual-polarisation operation of the horn antenna. Only one polarisation is considered for the analysis in this paper. This is established by exciting a single pair of pins while the remaining pair is terminated, thereby forming a twinax-feed.

There are a few considerations that need to be taken into account when designing the feed. First of all, each pin should be placed as close as possible to the inner edge of the corresponding ridge to minimise the excitation of higher-order modes. Secondly, the pin diameter and spacing, which determine the twinaxial line impedances, and the ridge thickness and gap width, which determine the wave impedances, needs to be matched in such a way that an optimum transition is created from the transmission line to the quad-ridged circular waveguide. Furthermore, another parameter that influences the 


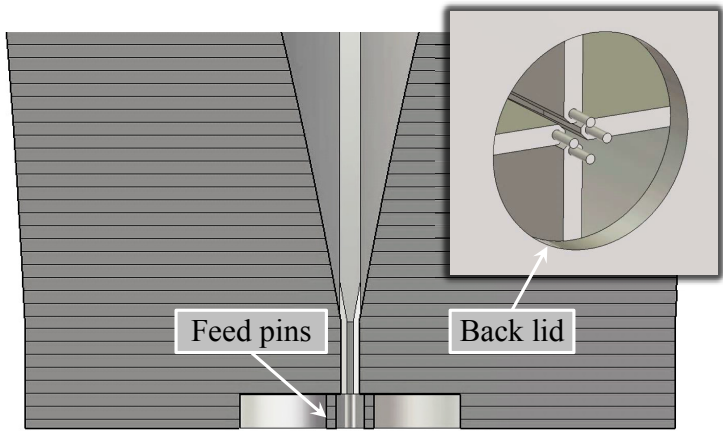

(a)

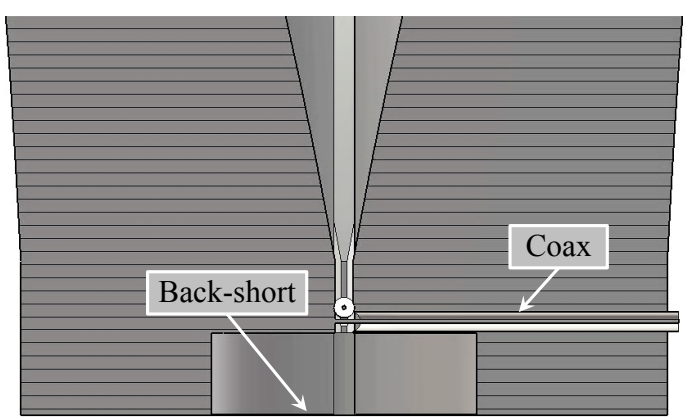

(b)

Fig. 2. CAD views of the cross-sections of two feed networks for the QRFH antenna in Fig. 1. (a) The quadraxial feed with centre conductors connected to the ridges, with the inset showing the back of the QRFH. (b) The conventional feed with a back-short section and ridge-to-coax transition.

differential impedance of the twinaxial line is the diameter of the cylinder in the back lid of the QRFH, shown in Fig.2(a). This diameter should be small enough in order for the higherorder modes to be below cut-off in the band of operation.

A unique feature of the QRFH antenna is that it can easily be separated into two independent sections as indicated in Fig. 1, reducing simulation time for design purposes. Here the throat section refers to the uniform waveguide terminated by the feeding network, while the flared section is the radiating part of the antenna. The flared section therefore serves as a good matching network between free-space and the fundamental $T E_{11}$ mode in the throat.

In order to compare the performance of the proposed feed with the conventional balun consisting of a back-short section and ridge-to-coax transition as shown in Fig. 2(b), a QRFH antenna operating from 2 to $12 \mathrm{GHz}$ is designed. The values of the throat dimensions (i.e. cylinder diameter, ridge thickness, gap width, etc.) are taken from an existing QRFH design for a coax-feed with a characteristic impedance of $100 \Omega$. The dimensions of the flared section (i.e. horn length, aperture size and taper) are optimised for constant beamwidth over the frequency range, while the horn is excited with a pure $T E_{11}$ mode. The product is an antenna capable of being fed by either the coax- or twinax-feed.

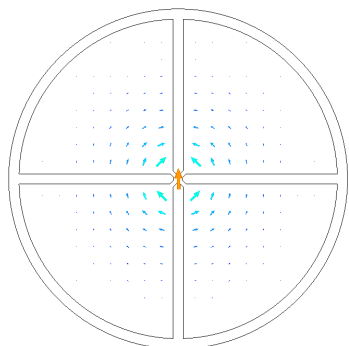

(a) $T E_{11}$

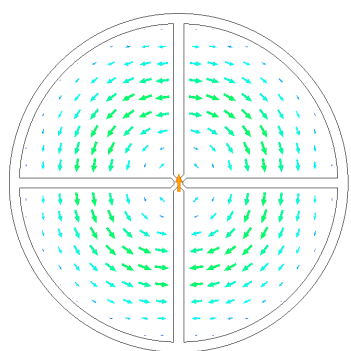

(c) $T E_{12}$

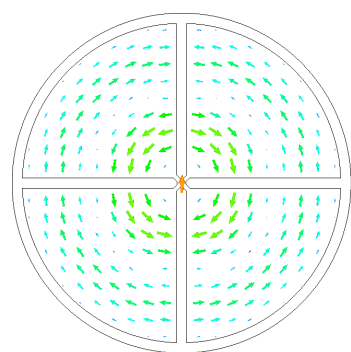

(e) $T E_{13}$

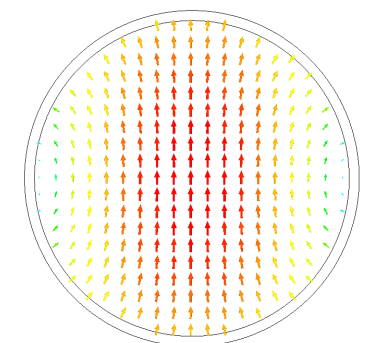

(b) $T E_{11}$

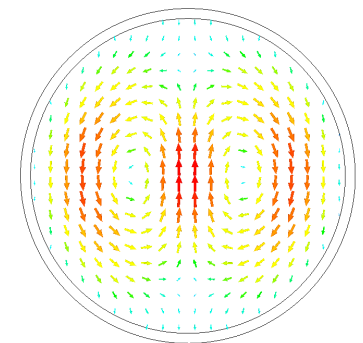

(d) $T E_{12}$

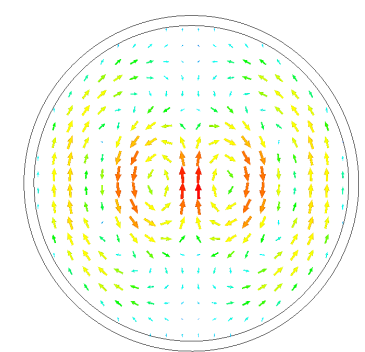

(f) $T E_{13}$
Fig. 3. The E-field distributions of the $T E_{11}, T E_{12}$ and $T E_{13}$ modes in circular waveguides with and without ridges.

\section{Modal CONTEnt AnAlysis}

As illustrated in [1] through a simplified analytic approach, the $T E_{11}$ mode should be dominant in the aperture field distribution of a QRFH over a large bandwidth, in order to achieve the required radiation. The subordinate modes should be in declining order of power $T M_{11}, T E_{12}, T M_{12}, T E_{13}$, etc. In Fig. 3 the field distributions of the first three $T E$ modes are shown for clarity. Furthermore, due to the twofold symmetry of the aperture field distribution, all evenorder modes are undesired (i.e. $T E_{m n}$ and $T M_{m n}$ where $m=0,2,4, \ldots)$.

In this work, the modal content in the throat of the QRFH antenna is investigated through full-wave simulations using Computer Simulation Technology's Microwave Suite software (CST-MWS). For this purpose a waveguide port is placed at the plane where the flared section of the complete QRFH model would begin. The simulated S-parameters of the throat section with either a coax- or twinax-feed are shown in Fig. 4(a) and 4(b), respectively. These results only include the significant excited modes.

As expected, the $T E_{11}$ mode is most strongly excited over 

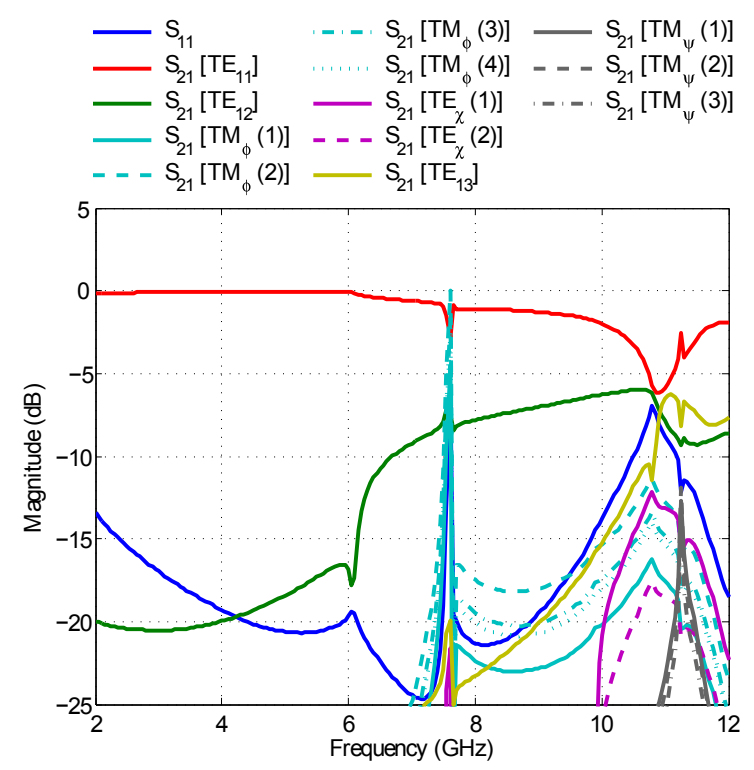

(a)

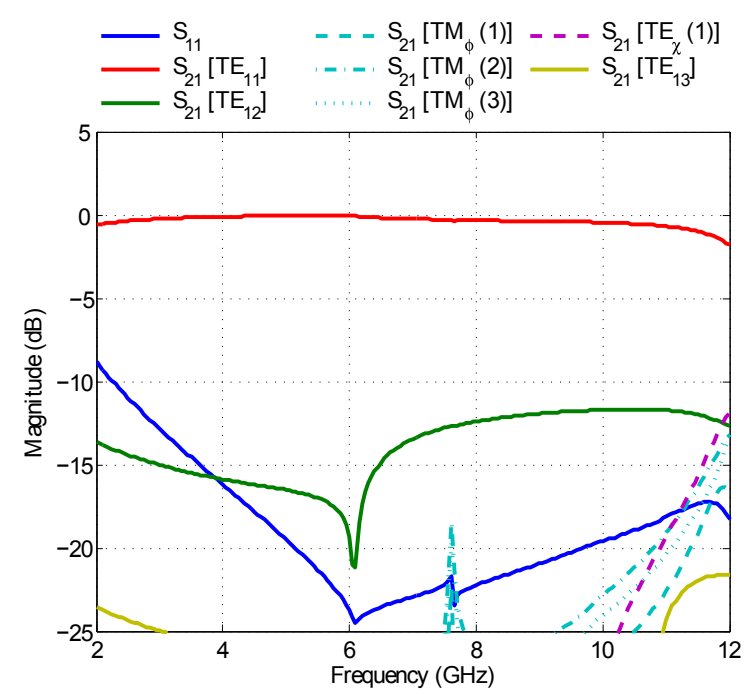

(b)

Fig. 4. The simulated S-parameters of the throat section of the QRFH, excited by either the (a) coax- or (b) twinax-feed. In both these configurations port 1 is connected to the feed terminals while port 2 is a waveguide port terminating the quad-ridged circular waveguide. The following modes are representative of a superposition of cylindrical modes: $\left\{T M_{01}, T M_{11}, T M_{21 L}\right\} \subset T M_{\phi}$; $\left\{T E_{22 U}, T E_{32}, T E_{42 L}\right\} \subset T E_{\chi} ;\left\{T M_{21 U}, T M_{12}, T M_{41 L}\right\} \subset T M_{\psi}$. Note that the notations $U$ and $L$ refer to the upper and lower modes, respectively.

the wide frequency band $\left(\left|S_{2,1}\left[T E_{11}\right]\right|\right.$ is close to $\left.0 \mathrm{~dB}\right)$ for both feeds, whereas the contents of the higher-order modes are rather different. Interestingly enough, the second strongest mode is $T E_{12}$ and is excited significantly higher with the coax-feed than with the twinax-feed for the majority of the frequency band. Although this mode is one of the desired subordinate aperture modes, its presence in the throat section is not beneficial. The purpose of the flared section is to excite the higher-order modes required in the aperture from the dominant
$T E_{11}$ mode. Consequently with the $T E_{12}$ mode present in the throat, unwanted modal distributions are obtained in the aperture. Furthermore, the reason for $\left|S_{2,1}\left[T E_{12}\right]\right|$ being larger at the lower frequencies in Fig. 4(b), is due to a slight imbalance of the energy in the simulation. Note that this mode is evanescent below $6.1 \mathrm{GHz}$.

Another important observation is that the $T E_{13}$ mode has a cut-off frequency at $10.8 \mathrm{GHz}$ which directly relates to the strong resonance in Fig. 4(a), causing a significant drop in $\left|S_{2,1}\right|$ of the fundamental $T E_{11}$ mode. This phenomenon is not present with the twinax-feed due to the weak excitation of $T E_{13}$ and hence, $T E_{11}$ is unchanged over the frequency range in Fig. 4(b). Note that in a similar manner does the peak at $7.6 \mathrm{GHz}$ relate to the cut-off frequency of $T M_{\phi}$.

The $T M_{\phi}, T E_{\chi}$ and $T M_{\psi}$ modes refer to combinations of modes. In ridged circular waveguides the cut-off frequencies of specific cylindrical modes tend to coincide as the gap narrows between the ridges. CST-MWS calculates the field distributions as a superposition of these types of modes according to its own criteria and not necessarily based on the original cylindrical modes. In Fig. 4, the $T M_{\phi}$ modes represent a superposition of $T M_{01}, T M_{11}$ and $T M_{21 L}$; while the $T E_{\chi}$ modes represent $T E_{22 U}, T E_{32}$ and $T E_{42 L}$; and $T M_{\psi}$ modes represent $T M_{21 U}, T M_{12}$ and $T M_{41 L}$. Note that the notations $U$ and $L$ refer to the upper and lower modes, respectively, which is the result of mode-splitting due to the inductive and capacitive loading of the ridges [6]. The numerical notations in Fig. 4 refer to the degenerate modes [e.g. with $T M_{\phi}(1)-(4)$ ].

It is clearly seen that these higher-order modes are strongly excited in the throat with the coax-feed, while the twinaxfeed substantially suppresses all of them. The effects of these higher-order modes are seen in the far-field performance of the QRFH, presented in Section IV.

\section{FAR-FIELD PERFORMANCE}

Although a quadruple-ridged flared horn is often presented in the literature as a (near-) constant beamwidth antenna with about $6: 1$ bandwidth [1], its beamwidth in the H-plane (typically defined at the $-10 \mathrm{~dB}$ level) varies with as much as a factor of 3 over the frequency band. This strong variation results from rapid narrowing in the beam at high frequencies, where the contribution of the high-order modes launched by the coaxial feed is comparable to that of the fundamental $T E_{11}$ mode. This detrimental effect of the coaxial feed excitation cannot be corrected by standard (narrowband) mode suppression techniques. For example, a metal ring structure, which was introduced in the design of a QRFH in [7] in order to suppress the $T E_{12}$ mode in the throat section, has been shown to improve the E-plane beam performance but not the beam in the H-plane.

To illustrate the expected advantages of the proposed wideband solution, Figures 6 and 7 compare the pattern cuts in the $\mathrm{E}, \mathrm{H}$ and $45^{\circ}$ planes for the present design of the QRFH antenna when it is fed with either the coaxial or the twinaxial feed. Each graph presents the results of seven frequency points 
ranging from 6 to $12 \mathrm{GHz}$ for a single polarisation, with the cross-polarisation cuts depicted by the dashed lines. The results at lower frequencies are omitted due to their similarity, as is expected following the modal analysis in Section III.

As one can see, the far-field patterns of the coax-fed horn exhibit larger ripples, as compared to that of the twinax-fed horn, which is expected due the presence of strongly excited high-order modes in the throat section with the coax excitation [see Fig. 4(a)]. The asymmetry of this type of excitation is also observable in the far-fields, e.g. in Fig. 6(b) this effect is clearly seen from the radiation pattern cut at $11 \mathrm{GHz}$.

In general, the overall shape of the pattern cuts, their symmetry in all the elevation planes and frequency behavior over the band has been significantly improved with the proposed twinax-feed. The latter is evident from the $-10 \mathrm{~dB}$ beamwidth shown in Fig. 5(a) as a function of frequency over the entire 2 to $12 \mathrm{GHz}$ band. Furthermore, the cross-polarisation patterns [see Fig. 6(b) and Fig. 7(b)] show reduced peak levels at most frequencies from 6 to $12 \mathrm{GHz}$ for the case of the twinaxfeed, where the relative improvement is as large as $6 \mathrm{~dB}$. The frequency response of the normalized peak cross-polarisation over the entire band is shown in Fig. 5(b) and demonstrates a very good performance in comparison to the coax-fed horn for which high frequencies are problematic in achieving low cross-polarisation. This is in agreement with the results in [1] for a slightly different design of the coax-fed horn; the data from this work has been added to Fig. 5(b) to illustrate this resemblance.

\section{CONCLusion}

A new feed is proposed for the QRFH antenna, which allows the integration of dLNAs and presents the possibility of non-standard input impedances. In this work it is shown that the quadraxial feed improves the modal content in the throat section of a QRFH compared with the conventional coaxial feed, by strongly exciting the fundamental $T E_{11}$ mode while suppressing the unwanted higher-order modes. This feature does not only present more freedom in the design, but also significantly improves the antenna performance.

The simulated results of the QRFH antenna, excited with the quadraxial feed, shows improvement in the overall shape of the radiation patterns, their symmetry in the elevation planes and frequency behaviour over the ultra-wide 6:1 bandwidth. The cross-polarisation levels are also significantly reduced in comparison with the coaxial feed - with as much as $6 \mathrm{~dB}$ in the problematic region of the high frequencies.

\section{ACKNOWLEDGEMENT}

The authors would like to acknowledge the SKA South Africa and NRF South Africa as well as the Swedish VR and VINNOVA agencies, for funding this work.

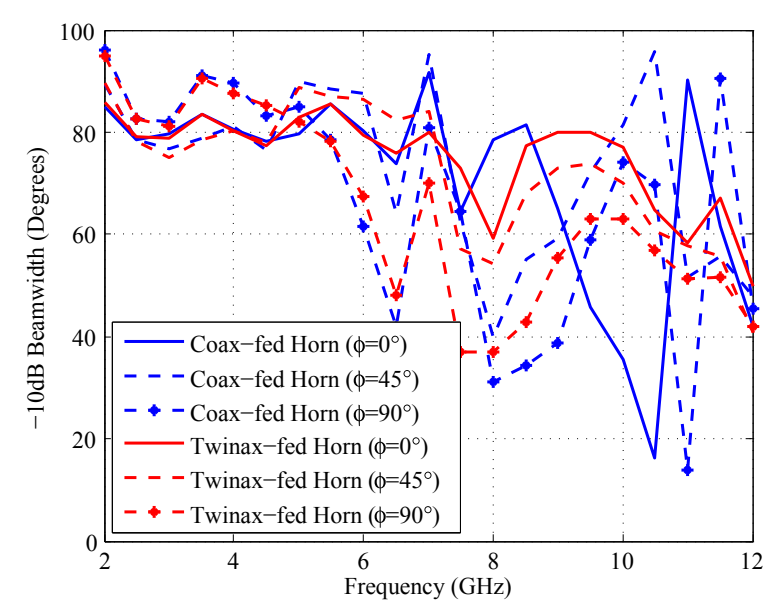

(a)

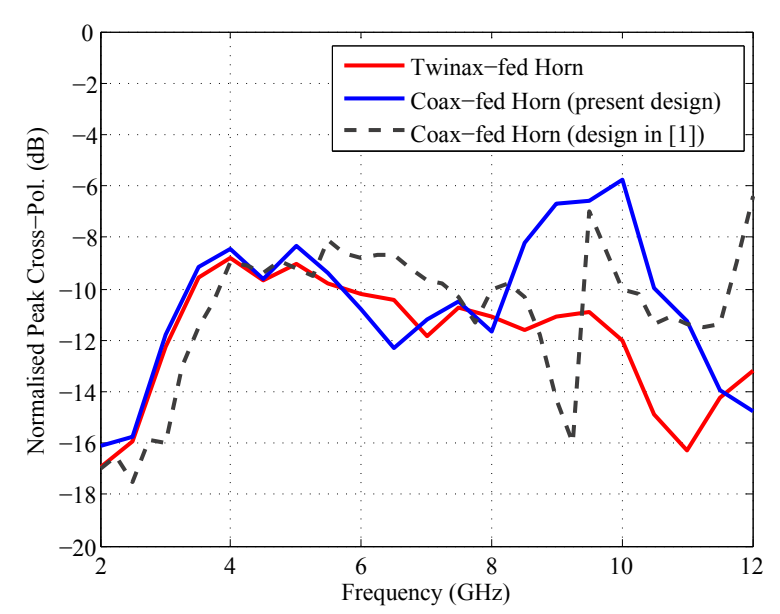

(b)

Fig. 5. The (a) $-10 \mathrm{~dB}$ beamwidths and (b) normalised peak cross-polarisation levels of the coax- and twinax-fed QRFH antennas.

\section{REFERENCES}

[1] A. Akgiray, S. Weinreb, W. Imbriale, and C. Beaudoin, "Circular quadruple-ridged flared horn achieving near-constant beamwidth over multioctave bandwidth: Design and measurements," IEEE Trans. Antennas Propag., vol. 61, no. 3, pp. 1099-1108, March 2013.

[2] J. Yang, M. Pantaleev, P.-S. Kildal, and L. Helldner, "Design of compact dual-polarized 1.2-10 GHz eleven feed for decade bandwidth radio telescopes," IEEE Trans. Antennas Propag., vol. 60, no. 5, pp. 22102218, May 2012.

[3] R. Gawande and R. Bradley, "Towards an ultra wideband low noise active sinuous feed for next generation radio telescopes," IEEE Trans. Antennas Propag., vol. 59, no. 6, pp. 1945-1953, June 2011.

[4] G. Cortes-Medellin, "Non-planar quasi-self-complementary ultrawideband feed antenna," IEEE Trans. Antennas Propag., vol. 59, no. 6, pp. 1935-1944, June 2011.

[5] M. Ivashina, R. Maaskant, and B. Woestenburg, "Equivalent system representation to model the beam sensitivity of receiving antenna arrays," IEEE Antennas Wireless Propag. Lett., vol. 7, pp. 733-737, October 2008.

[6] M. Chen, G. Tsandoulas, and F. Willwerth, "Modal characteristics of quadruple-ridged circular and square waveguides," IEEE Trans. Microw. Theory Techn., vol. 22, no. 8, pp. 801-804, 1974.

[7] A. Akgiray, S. Weinreb, and W. Imbriale, "Design and measurements of dual-polarized wideband constant-beamwidth quadruple-ridged flared horn," in Proc. IEEE Antennas Propag. Soc. Int. Symp., 2011, pp. 11351138 . 


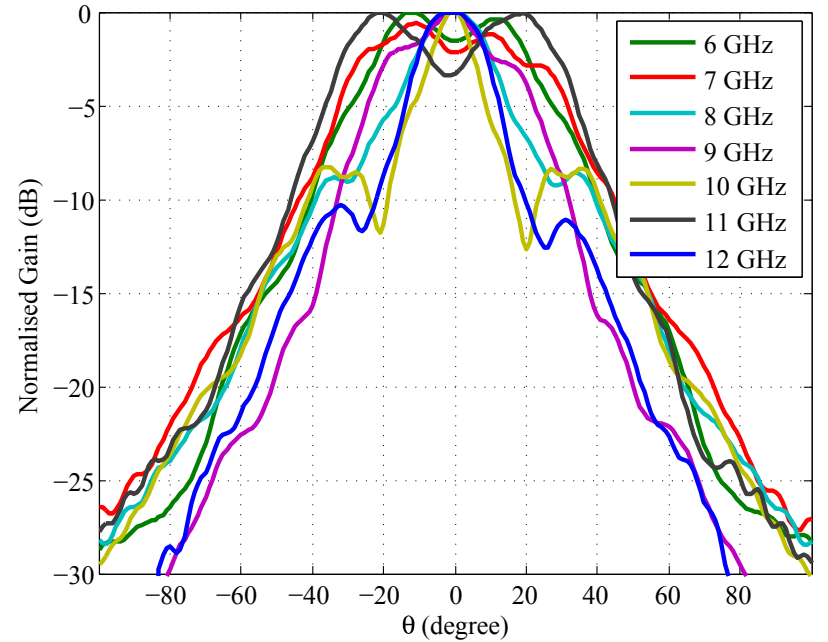

(a)

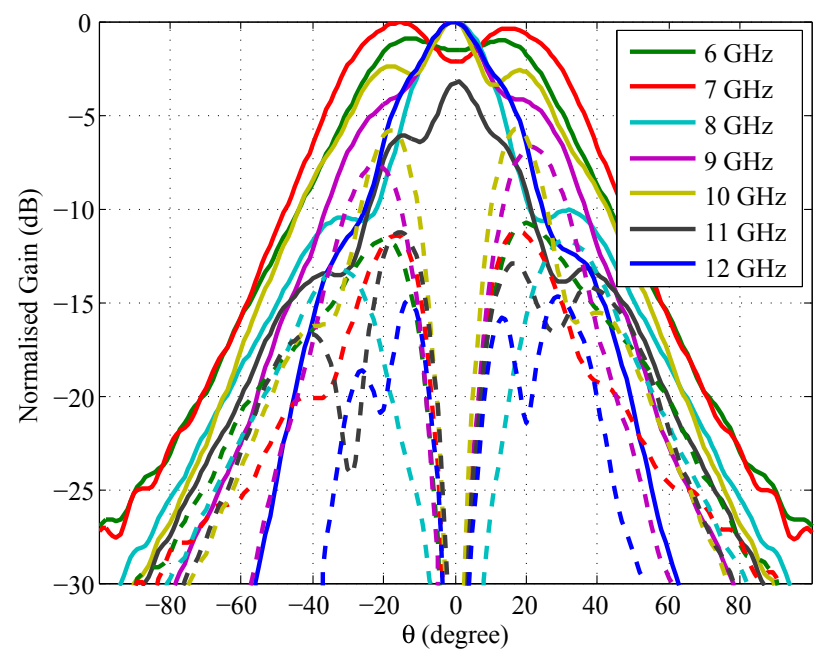

(b)

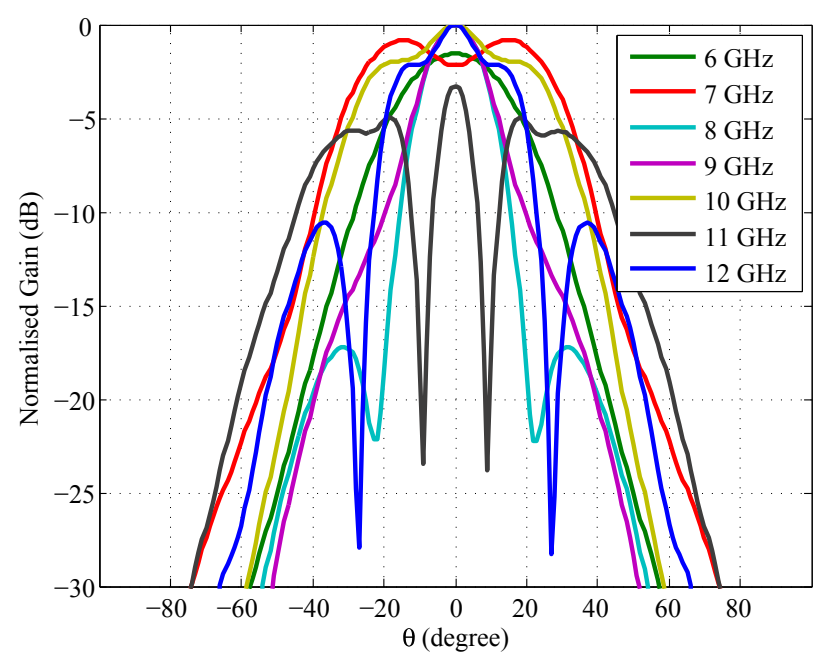

(c)

Fig. 6. The far-field patterns of the coax-fed QRFH antenna in the (a) Eplane, (b) D-plane and (c) H-plane. The cross-polarisations are depicted by the dashed lines.

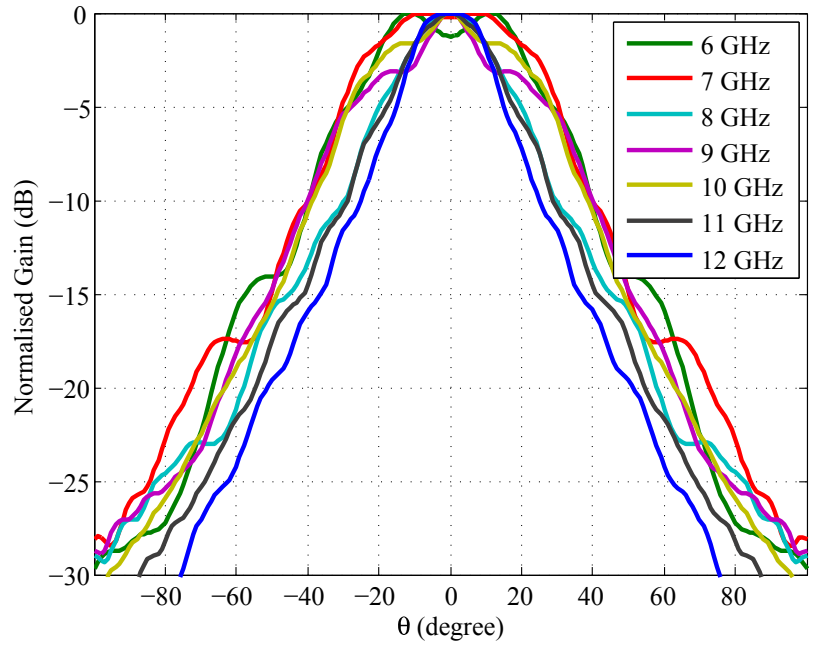

(a)

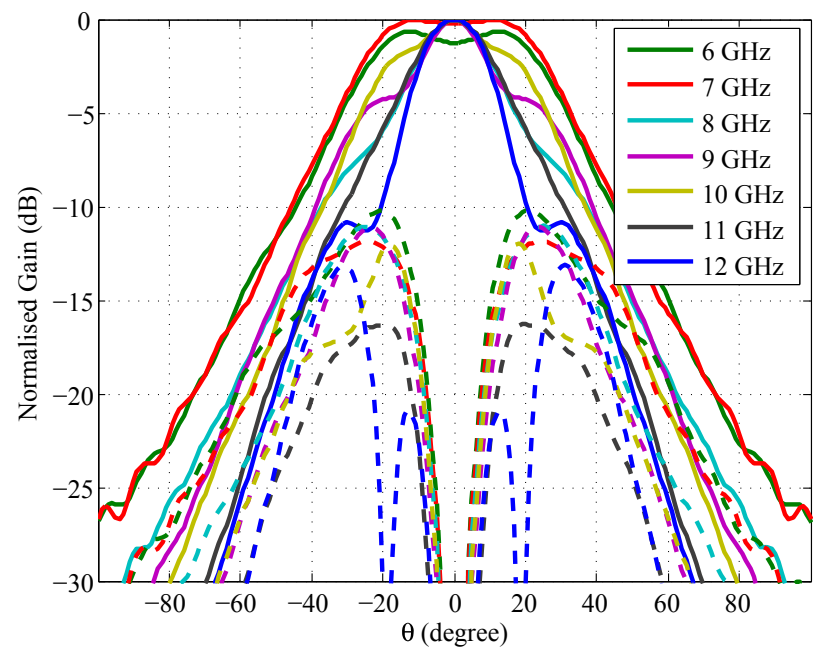

(b)

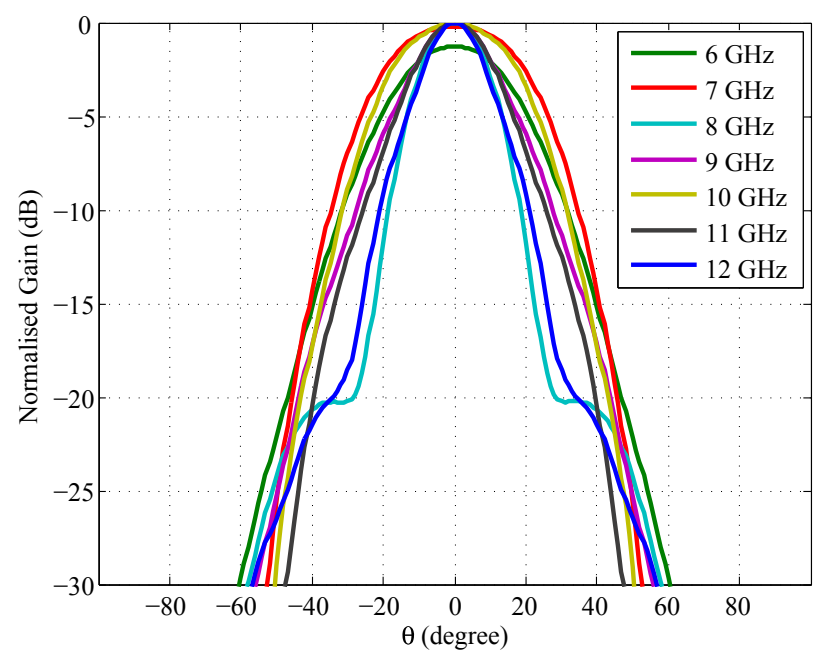

(c)

Fig. 7. The far-field patterns of the twinax-fed QRFH antenna in the (a) Eplane, (b) D-plane and (c) H-plane. The cross-polarisations are depicted by the dashed lines. 